\title{
Oil spill detection system: results from field trials October 2004
}

\author{
R. Gangeskar \& E. Nøst \\ Department of Research and Development, Miros AS, Norway
}

\begin{abstract}
Oil spill incidents represent a large cost, economically and environmentally. It is of great importance to detect an oil spill as soon as possible after a leakage, in order to reduce the consequences. Wavex is a system for monitoring scaled directional wave spectra and sea surface currents from X-band radar images. The oil spill detection (OSD) system considered in this paper consists of the hardware and software modules of the Wavex system, in addition to software modules specific for oil detection purposes. The principle of measurement is based on the fact that areas covered by oil spill reflect less microwave power, due to the dampening of the sea surface capillary waves. The OSD system was thoroughly tested at a field trial carried out in October 2004 near the Troll field, off the west coast of Norway. The Norwegian Clean Seas Association for Operating Companies (NOFO) especially arranged the trial for this purpose. Six small releases of oil equivalents were discharged, and they could be detected and followed by the system during various wind and wave conditions. Images providing an average of the back-scattered intensity from various parts of the sea surface were shown to be useful for the system operator. Continuous surveillance of local areas is particularly helpful during the hours of darkness. Later, the data were reprocessed with a new and completely automatic oil detection algorithm using methods of image segmentation and object classification, and the oil was automatically detected. This algorithm is now implemented in the real-time system. More effort should be put into testing the system operation during marginal conditions, such as low wind speeds with non-optimal directions of sight and rougher sea states, as well as testing the system during various geometrical conditions.
\end{abstract}

Keywords: oil spill detection, field trials, navigation radar, signal processing, image processing. 


\section{Introduction}

It is of great importance to detect an oil spill as soon as possible after a leakage, in order to reduce the environmental and economical consequences. The oil spill detection (OSD) system tested in the trials described in this work has until now shown to be a promising and helpful tool for such incidents, both during cleanup operations of already discovered oil and for the detection of new spills. The system extracts information regarding possible oil on the sea surface from Xband radar images. This implies the possibility of continuous surveillance of local areas of particular interest, also during the hours of darkness.

The OSD system was thoroughly tested through field trials at sea nearby the Troll field off the west coast of Norway in October 2004. Results from these trials are presented here. The Norwegian Clean Seas Association for Operating Companies (NOFO) organised the trials especially for testing this system.

\subsection{The objective of the field trials}

The main objective of the field trials for the OSD system was (Rødal [1]): "To verify that the ship-based radar system continuously, and in approximately realtime, can determine the location and extent of oil on the sea surface within a radius of 1 nautical mile or more in sea states corresponding to Beaufort 2-6".

\subsection{Description of the oil spill detection system}

Wavex (Grønlie [2]) is a system for monitoring scaled directional wave spectra and sea surface currents from X-band radar images. The OSD system consists of the hardware and software modules of the Wavex system, in addition to software modules specific for oil detection purposes. By means of the Wavex system hardware, digitised X-band radar sea surface images are collected. These images are further processed to obtain scaled directional wave spectra, sea surface currents and images indicating any oil spill. In addition, other environmental data, such as wind, are collected by the system and used for quality control.

The principle of oil detection is based on the fact that areas covered by oil reflect less microwave power, due to the dampening of the sea surface capillary waves. This causes oil to generate darker areas in the radar images. The algorithms (Gangeskar [3]) contain functionality for making these areas appear rather distinctly in back-scatter intensity (BSI) images, which are images providing information about the average back-scattered electromagnetic intensity from the various areas of the sea surface. Further, the algorithms include functionality for automatic oil detection and tracking based on the information in the BSI images. The output of the automatic detection is oil spill (OS) images indicating any areas of the sea surface covered by oil. These OS images are binary in the sense that each image pixel has the value of water or oil.

The system cannot produce reasonable results when the wind speed is below approximately $2 \mathrm{~m} / \mathrm{s}$, due to lack of capillary waves. This lower wind speed limit is consistent with findings from other trials (Benelli and Garzelli [4]). The 
system does not include any specific functionality for discriminating between oil on the sea surface and other phenomena causing the dampening of the capillary waves, such as grease ice, current shear zones, or organic films due to algae bloom. The system does, however, include a tracking functionality verifying that an image area appearing as oil spill actually moves like a real oil spill a few minutes before triggering an oil spill alarm.

The algorithms used for oil detection during these trials were developed using data from the Deep Spill trial onboard the vessel Johan Hjort in June 2000. The algorithms are described at various stages during the development by Gangeskar [3][5],[6]].

The Wavex system was installed on Skandi Admiral a few weeks prior to the trials (Karoliussen [7]). It should be noted that the radar antenna height above the sea surface was $28 \mathrm{~m}$, and that oil detection astern was not possible due to a blind area of 80 degrees caused by shadowing from masts.

\section{The field trials}

The OSD system was tested during the $10^{\text {th }}$ and $11^{\text {th }}$ of October 2004 and compared with the performance of other surveillance systems. Six oil spills totalling only $1 \mathrm{~m}^{3}$ of oil were discharged during the field trials.

During the tests the vessel Skandi Admiral, a surveillance aeroplane, a helicopter from CHC Helicopter Service and the satellite RADARSAT 1 were all used to observe and survey the oil spills. In addition to the OSD system on Skandi Admiral, an aerostat with FLIR (an airborne craft anchored to Skandi Admiral and filled with helium) and a handhold IR camera were tested. In this work the results from the OSD system are considered, and these are briefly compared with digital photos from the surveillance aeroplane.

\subsection{The oil spills}

Detailed information concerning the oil spills and the wind situation, in addition to the vessel speed and track during the release of the spills are given in Table 1.

Spill number 1 contained 400 litres of diesel and was released the morning of the $10^{\text {th }}$ of October 2004. Spill number 2 contained 580 litres of emulsion with 200 litres of crude oil and was released about two hours later. An example of a digital photo taken from the aeroplane is shown in Figure 1. The succeeding spills, i.e. spills number 3-6, were all of 300 litres of emulsion with 100 litres of crude oil. Spill number 3 was released the $10^{\text {th }}$ of October 2004 and the spills number 4, 5 and 6 the next day.

During the release of spills number 1, 2 and 3, the significant wave height was below 2 metres and the wind speed was mostly below $3.5 \mathrm{~m} / \mathrm{s}$. Hence, the sea state during observation of these spills corresponds to Beaufort 2 (Andersen [8]). For spills number 4, 5 and 6 the wind speed varied between 5.5 and $8 \mathrm{~m} / \mathrm{s}$ and the significant wave height was approximately $2.5 \mathrm{~m}$, corresponding to a sea state within Beaufort 4. 
Table 1: Information concerning the oil spills.

\begin{tabular}{||l|l|l|l|l|l|l|l|l||}
\hline \multirow{2}{*}{$\begin{array}{l}\text { Spill } \\
\text { No. }\end{array}$} & \multirow{2}{*}{$\begin{array}{l}\text { Amount } \\
(\text { litres })\end{array}$} & \multirow{2}{*}{ Quality } & & \multicolumn{3}{l|}{ Pumping spill } & \multicolumn{3}{|l|}{ Average values during pumping } \\
\cline { 6 - 10 } & & & Date & $\begin{array}{l}\text { Time } \\
\text { finished }\end{array}$ & $\begin{array}{l}\text { Sessel } \\
(\mathrm{m} / \mathrm{s})\end{array}$ & $\begin{array}{l}\text { Track } \\
(\mathrm{deg})\end{array}$ & $\begin{array}{l}\text { Speed } \\
(\mathrm{m} / \mathrm{s})\end{array}$ & $\begin{array}{l}\text { Dir. } \\
(\mathrm{deg})\end{array}$ \\
\hline 1 & 400 & Diesel & $10^{\text {th }}$ & $06: 48$ & 1.2 & 233 & 4.0 & 342 \\
\hline 2 & 200 & Crude oil & $10^{\text {th }}$ & $08: 52$ & 0.7 & 276 & 2.7 & 58 \\
\hline 3 & 100 & Crude oil & $10^{\text {th }}$ & $14: 03$ & 0.1 & 143 & 3.8 & 316 \\
\hline 4 & 100 & Crude oil & $11^{\text {th }}$ & $05: 06$ & 0.9 & 7 & 6.2 & 205 \\
\hline 5 & 100 & Crude oil & $11^{\text {th }}$ & $06: 35$ & 0.8 & 106 & 6.5 & 199 \\
\hline 6 & 100 & Crude oil & $11^{\text {th }}$ & $06: 55$ & 0.8 & 83 & 7.1 & 188 \\
\hline
\end{tabular}

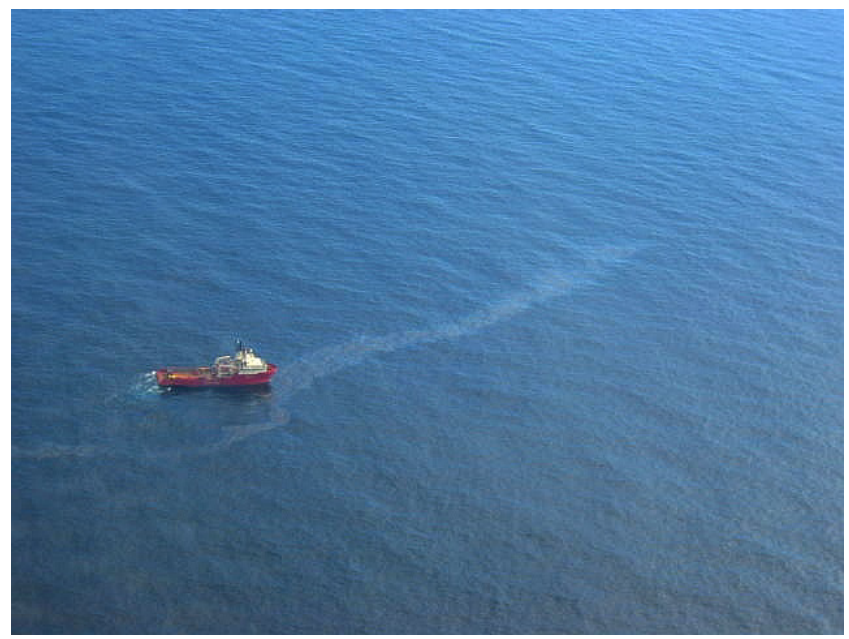

Figure 1: $\quad$ Photo from aeroplane of spill number 2.

\subsection{Data collected}

The OSD system processed the collected data and saved raw and processed data to historical files. Data from wind sensors, GPS and Gyro were processed and stored to files every minute, while series of raw image data from the Wavex system were collected with 3- and 4-minutes intervals and stored to files. In addition, processed data from the Wavex system, including scaled directional wave spectra and the sea surface current vector, were stored to files. The GPS data included the location, speed and track of the vessel. All OSD specific data, such as BSI images and OS images already mentioned, were stored to files in the same intervals as the Wavex data.

\section{Results of oil detections}

Results were obtained by the real-time system onboard Skandi Admiral and by the post-processing performed after the trials. 


\subsection{Real-time results}

The real-time results during the trials were documented by automatically generated screen dumps every 2 minutes. The back-scatter intensity (BSI) images and oil spill (OS) images always have the geographical north pointing upwards. The vessel sketch also indicates the vessel heading. The geographical position of the vessel is shown in the lower left corner of the BSI image.

As shown in Figure 2, the spills were released astern, as described by Rødal [1], in the blind area of the onboard radar. After turning the vessel, the oil spills were seen in the OSD displays.

Spill number 1 could be seen in the BSI images from UTC 06:52 to approximately 07:08, until 20 minutes after the diesel was released. During these 20 minutes the wind speed decreased from about $5.0 \mathrm{~m} / \mathrm{s}$ to $3.3 \mathrm{~m} / \mathrm{s}$. The diesel dissolved rather quickly.

Spill number 2 was clearly visible in the BSI images real-time during the trials, as shown in Figure 3. The location and extent of the oil spill seen in the BSI images comply well with photos taken from the aeroplane (Figure 1). The spill was visible for a period of 1 hour and 20 minutes after the release. During this period the wind speed first varied between 3 and $4 \mathrm{~m} / \mathrm{s}$, before decreasing to $2 \mathrm{~m} / \mathrm{s}$ and below. At UTC 10:15 when the wind speed was close to the lower limit of $2 \mathrm{~m} / \mathrm{s}$, the spill was hardly visible on the OSD display.

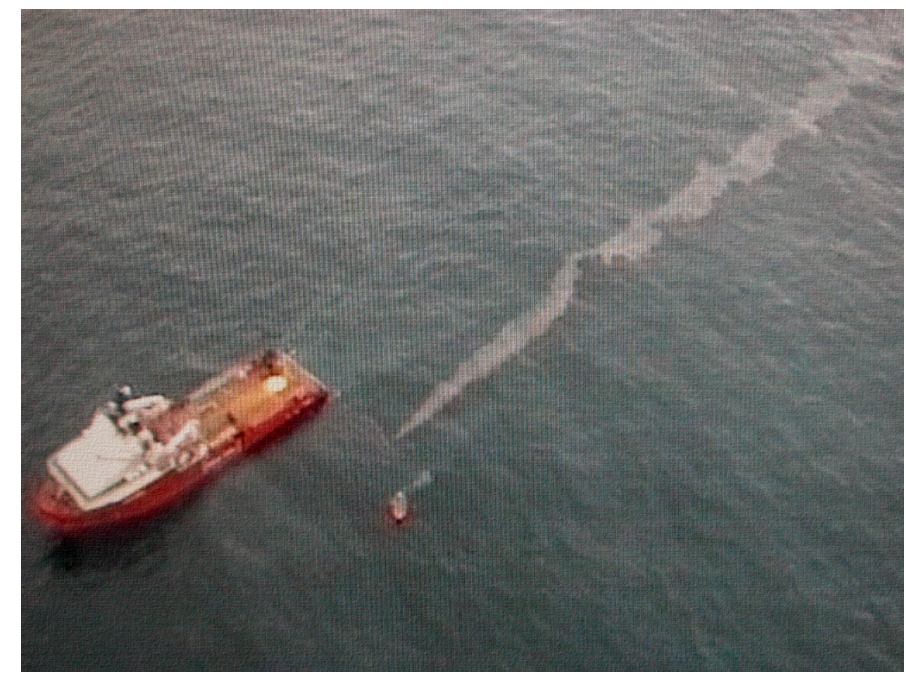

Figure 2: Release of oil spill from Skandi Admiral.

From UTC 10:15 until UTC 12:30 the wind speed was below $2 \mathrm{~m} / \mathrm{s}$, and the next spill release was postponed until a wind increase. At UTC 13:30 the wind speed had increased to about $4 \mathrm{~m} / \mathrm{s}$, and spill number 3 was initiated. However, the wind decreased quite fast and the spill was hardly seen on the OSD display. Areas of still water were observed from the bridge of Skandi Admiral, and the OSD system could not distinguish between areas of oil and areas of still water. 


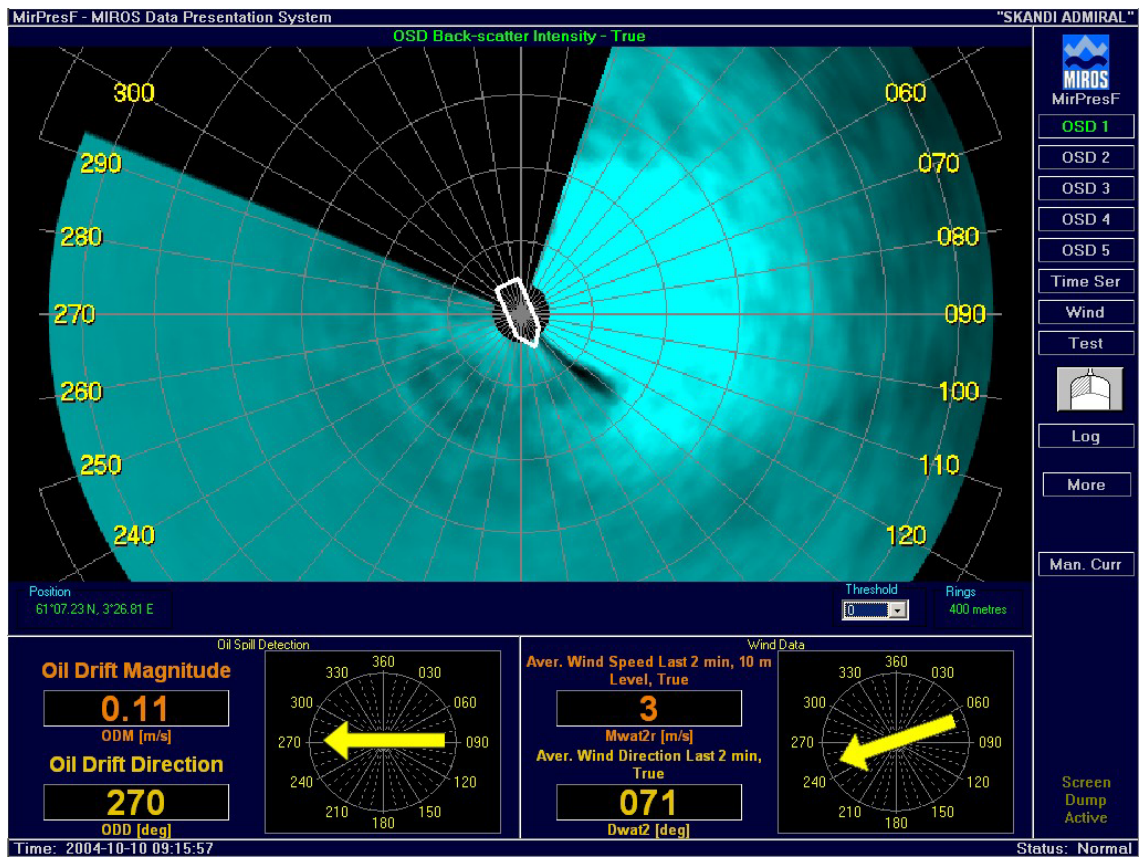

Figure 3: Real-time screen dump from spill no. 2, 20 minutes after release.

The following morning the wind situation had improved, with wind speeds between 5.5 and $8 \mathrm{~m} / \mathrm{s}$. Spill number 4 was released, and the oil was clearly visible in real-time in the OSD displays, as indicated by the dash circle in Figure 4. The BSI image of Figure 4 shows the 100 litres of crude oil 18 minutes after release, $0.7 \mathrm{~km}$ from the vessel. The spill has an extent of about 280 by 80 metres. This spill remained visible for 4 hours after it was released with conditions corresponding with Beaufort's scale 4 .

Spills number 5 and 6 were released 89 minutes and 109 minutes after spill number 4 respectively. All 3 spills could simultaneously be seen in the OSD display during the following hours. Figure 5 shows an example of such a display, 2 hours and 29 minutes after the release of spill number 4 .

\subsection{Results from post-processing}

A comprehensive post-processing of the collected data was performed to improve the algorithms for automatic oil spill detection and to investigate the possibilities for using the OSD system with the radar in medium-pulse mode.

\subsubsection{Short-pulse data}

As the previous sections show, oil is clearly visible in the BSI images. At the time of the trials the automatic oil detection algorithms used to generate OS images were not fully developed. Hence, reasonable OS images were not obtained in many real-time situations during the trials. After the trials the work 
on the algorithms was completed (Gangeskar [3]) and the data collected during the trials were reprocessed in order to test the automatic oil detection functionality. Reasonable OS images were obtained for all periods with reasonable BSI images. Figure 6 shows a screen dump of a series of automatic oil spill detections from spill number 4 . The most recent detection is indicated nearest to the vessel in the upper right of the display, and the 3 preceding detections are indicated at gradually increasing distances to the vessel. Note that the drift of the oil spill is clearly visible from the trace image, and that the drift is consistent both with the automatically estimated drift vector and with the wind direction in the lower right of the display.

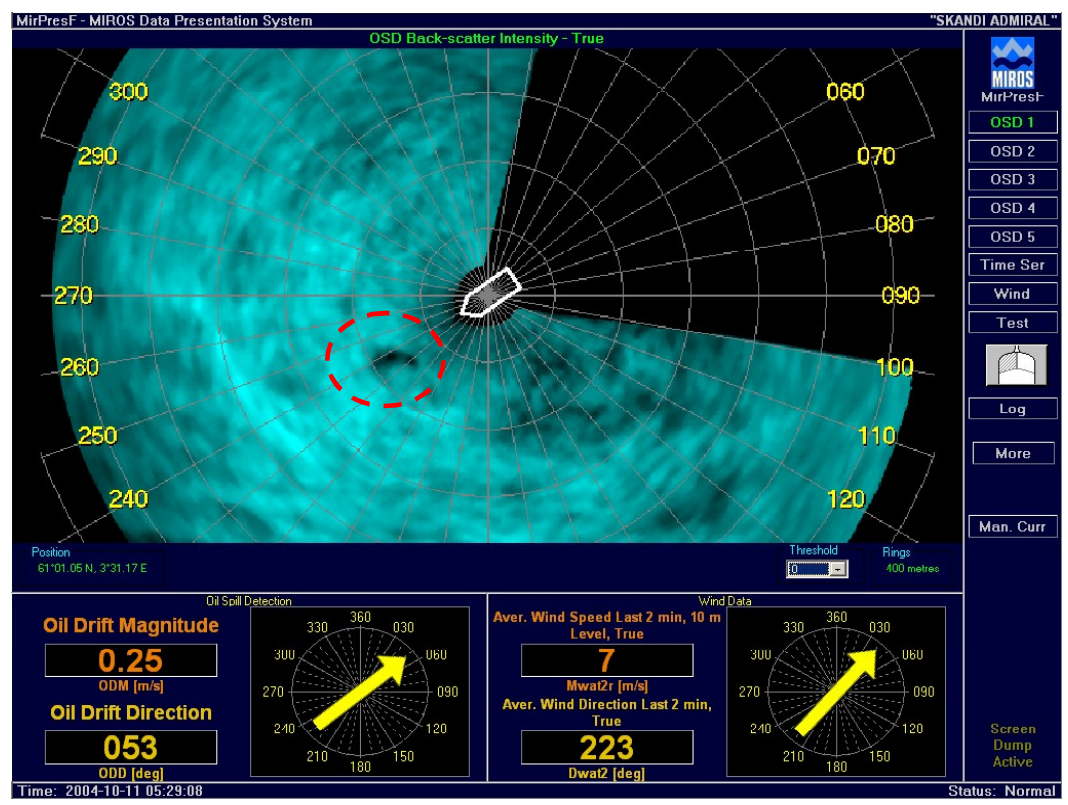

Figure 4: $\quad$ Real-time screen dump from spill no. 4.

\subsubsection{Medium-pulse data}

During the trials the radar was set to medium-pulse mode with a pulse width of $150 \mathrm{~ns}$ for 1 hour in order to collect data to be processed later. The default operating mode for the system has until now been short-pulse with a pulse width of $50 \mathrm{~ns}$, and this has limited the range to approximately $2.5 \mathrm{~km}$. The mediumpulse data were post-processed in order to inspect the possibilities for performing oil detection at greater ranges without increasing the radar antenna elevation.

Skandi Admiral first left the oil spills, and then turned and slowly approached the oil spills again. The BSI images obtained from this slowly approach have been inspected to determine the maximum range where the oil spills are visible.

Figure 7 shows a BSI image with oil spills number 4, 5 and 6 at a distance of $3.5 \mathrm{~km}$. The oil spills are clearly visible even without prior knowledge of their presence. The oil can be followed at distances of approximately $4.5 \mathrm{~km}$ as long 
as their presence and location are known. The spills totally disappear in the BSI images at distances greater than $5 \mathrm{~km}$. It is, however, not possible to conclude that $5 \mathrm{~km}$ is an absolute range limit for the system based on these trials. It is still possible that an increase in the oil volume, an increase in the antenna height or maybe a newer radar magnetron could make oil spills visible at greater distances. It should also be noted that a few degrees of dithering in the azimuth dimension of the radar images were present during the trials due to inadequate quality of the azimuth pulses from the radar. This caused image information to be smeared out, and thereby making oil detection more difficult, particularly at long ranges.

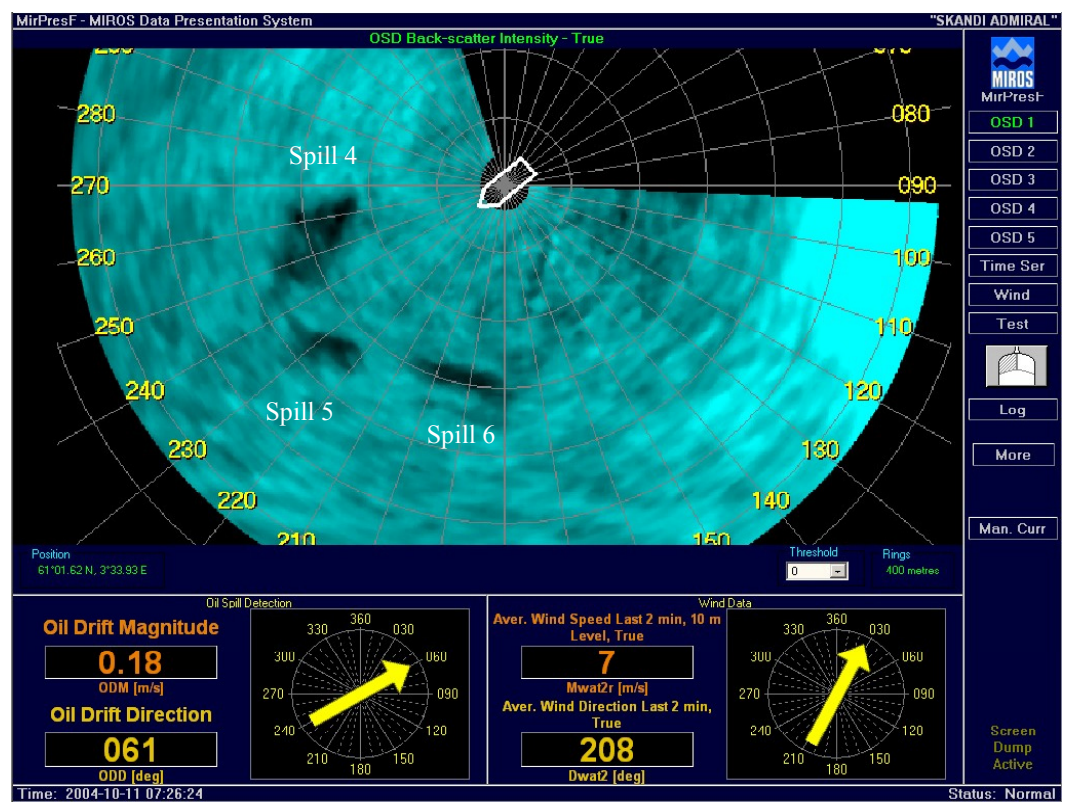

Figure 5: $\quad$ Real-time screen dump showing spill no. 4, 5 and 6.

\section{Summary and conclusions}

The oil spill detection (OSD) system produced a large selection of reliable realtime results during the trials. The post-processing also brought about interesting results. It was verified that small amounts of oil can be manually detected in realtime during day- and night-time by inspecting the back-scatter intensity (BSI) images generated by the system. In addition, the algorithms used to estimate the BSI images and to automatically detect oil spill were proven to provide results fulfilling the main objective of the field trials in Beaufort's scale 2 and 4.

100 litres of crude oil remained visible 4 hours after the release in conditions corresponding with Beaufort's scale 4. Small amounts of oil were clearly visible in BSI images up to distances of $3.5 \mathrm{~km}$ when using the radar in medium-pulse mode. 


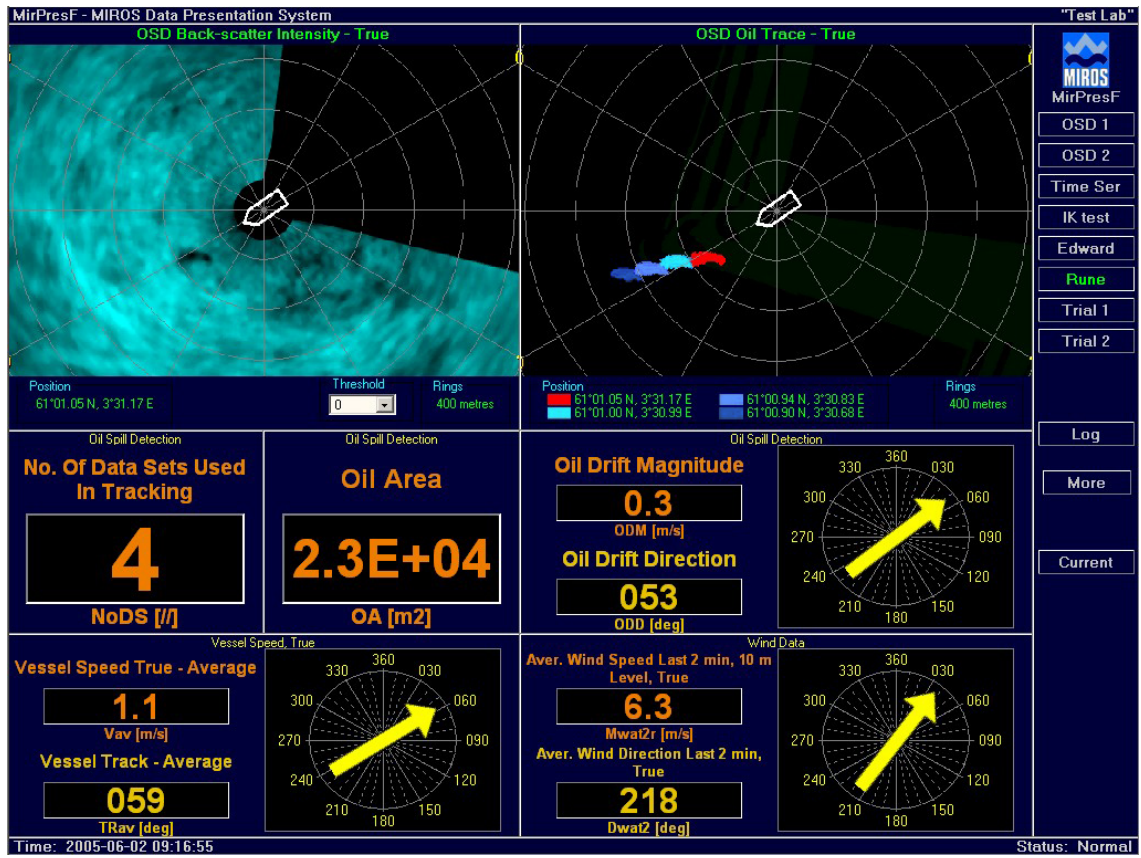

Figure 6: $\quad$ Automatic detections of spill no. 4.

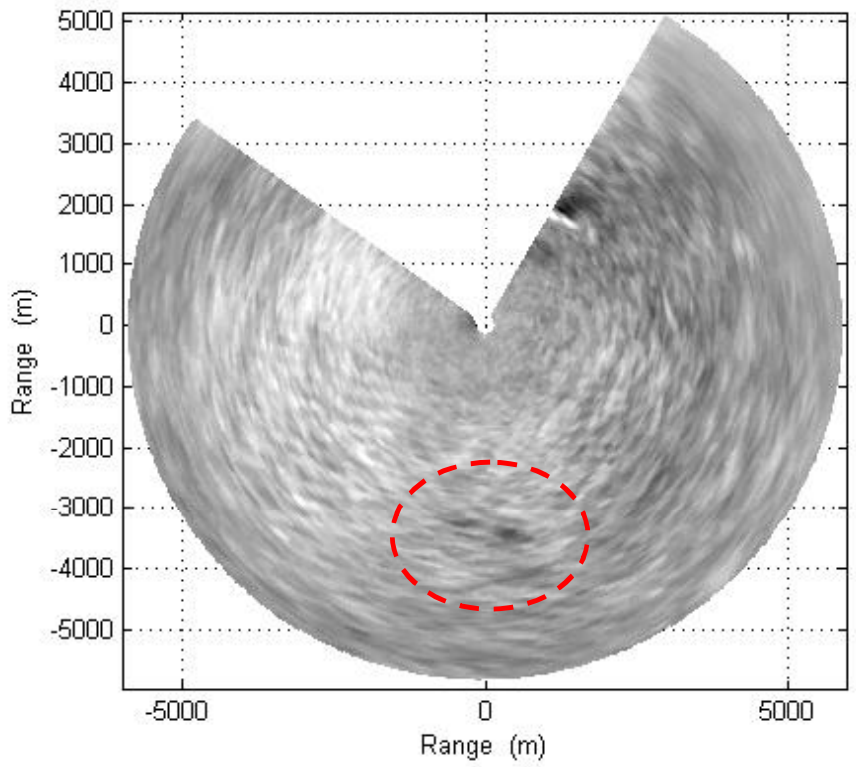

Figure 7: $\quad$ BSI image of medium-pulse data. 
It was verified that the OSD system hardware and software perform a continuous and stable operation, both with respect to real-time use and with respect to continuous storage of data required for post-processing.

The lower wind speed limit of $2 \mathrm{~m} / \mathrm{s}$ for reliable system operation was verified.

Future trials should preferably include testing during weather conditions with higher wind speeds and waves, in addition to higher antenna elevations and larger amounts of oil.

\section{References}

[1] Rødal, J., Operasjonsordre - Utprøving av oljedetekterende radar og håndholdt IR utstyr, NOFO, Norway, 9. October 2004.

[2] Grønlie, Ø., Wavex - Principles of Operation, Miros document, No. 1300/DD/011, Asker, Norway, 10. February 2004.

[3] Gangeskar, R., Automatic Oil Spill Detection by Marine X-Band Navigation Radar, Submitted to IEE Proc. Radar, Sonar \& Navigation, October 2005.

[4] Benelli, G. \& Garzelli, A., A multi-resolution approach to oil-spills detection in ERS-1 SAR images, part of the EUROPTO Conference on Image and Signal Processing for Remote Sensing, Barcelona, Spain, September 1998.

[5] Gangeskar, R., MIROS WAVEX Oil Spill Detection System - New Algorithm Tested on Data from Field Trials, Miros document, No. 3203/TN/001, Asker Norway, 21. May 2002.

[6] Gangeskar, R., Tuning of the OSD algorithm, Miros document, No. 3203/TN/002, Asker Norway, 7. June 2004.

[7] Karoliussen, I., "SKANDI ADMIRAL" SITE SURVEY, Miros Document, No. 3203/SR/001, Asker, Norway, 14. June 2004.

[8] Andersen, J. H. S., Oljedetekterende radar om bord supplyfartøy - Mål, delmål og funksjonskrav, Report number 4005100-150, NOFO, Norway, 15. March 2004. 